\title{
COGNITIVE DISTORTIONS AND EMOTION REGULATION AMONG POST TRAUMATIC STRESS DISORDER VICTIMS
}

\author{
Nawal Ouhmad ${ }^{1}$, Nicolas Combalbert ${ }^{1}$, \& Wissam El-Hage ${ }^{2}$ \\ ${ }^{1}$ Laboratory PAVEA, University of Tours (France) \\ ${ }^{2}$ Psychiatric Clinic, Saint Cyr sur Loire (France)
}

\begin{abstract}
Many authors have studied the links between cognition and emotions, including the links between cognitive distortions and emotional cognitive regulation strategies. Nevertheless, the explorations of these two instances in a clinical population presenting a posttraumatic stress disorder have very little been explored. The literature on this subject suggests that a specific profile could emerge in these subjects compared to a different clinical population and / or non-clinical. 184 subjects divided into three groups (60 subjects exposed to a traumatic event with PTSD, 61 subjects exposed to a traumatic event without PTSD, 63 control subjects) passed the CERQ, PCL-5 and a cognitive distortions scale.

The main results, which are preliminary results. highlight the presence of a profile distinguishing subjects with PTSD. They have difficulties in managing their emotions, as well as increased use of cognitive distortions, but they do not use them the most. The results lead us to question the cognitive functioning of people with PTSD but also the importance and consequences of therapeutic and drug monitoring of these patients.
\end{abstract}

Keywords: Cognitive distortions, cognitive emotional regulation, psychic trauma, posttraumatic stress disorder.

\section{Introduction}

Post-Traumatic stress disorder (PTSD) is a psychopathological disorder that is characterized by "the development of specific symptoms following exposure to a traumatic event in the context of death, death threats, serious injury or sexual assault "(WHO, 2016). Since 2013, PTSD has been classified as a "trauma and stress disorder" in the Diagnostic and Statistical Manual of Mental Disorders, Fifth Edition (DSM-5). PTSD is characterized by the appearance of psychiatric symptoms after exposure to one or more traumatic events. Generally, the diagnosis of PTSD is made after the month following the traumatic event (American Psychiatric Association, DSM 5, 2013). When an individual experience or witnesses a traumatic event, he may experience intense fear, helplessness or horror. Following this, a series of symptoms may appear, like cognitive distortions or difficulties to regular our emotions.

Beck $(1963,1964)$ defines cognitive distortions as fallacious reasoning that plays a determining role in the emergence of a number of mental disorders. Franceschi (2007) clarified the conceptual framework of cognitive distortions, distinguishing three key notions. The reference class consists of a set of phenomena or objects, for example, the reference class is composed of the character traits of the patient. The notion of duality, which corresponds to a pair of concepts such as positive / negative, internal / external, collective / individual, etc. In concrete terms, this refers to a criterion from which the elements of the reference class can be evaluated. We can note by A / $\bar{A}$ a given duality or A and $\bar{A}$ for dual concepts. And the taxon system. The taxonomy is a series of elements forming lists which concerns a domain. This taxonomy allows the patient to evaluate and classify the reference class according to criteria corresponds to a given duality A / $\overline{\mathrm{A}}$.

Regarding regulation of emotions, James Gross (1998) reintroduces the concept of emotional regulation into the field of cognitive science. The regulation of emotions refers to "the modulation of current emotional responses through a feedback loop on these responses" (Oschsner \& Gross, 2005). This refers to the set of strategies used by an individual to reduce, maintain or increase emotion or emotional responses. Garnefski et al. (2001) developed a scale to measure the preferred use of nine cognitive regulation strategies for emotions. 
Adaptive strategies group together several domains. Acceptance, when the individual expresses thoughts of acceptance and resignation in the face of the lived event. Positive concentration, focusing on more joyous and pleasant thoughts to deal with the lived event while concentration on the action is the concentration on the steps to follow to effectively manage the lived event. The positive reassessment will be when the individual will determine a positive sense of the event lived and will draw positive conclusions, finally, putting into perspective that is the fact of minimizing the seriousness of the event lived and highlighting its relative relativity. to other events considered more serious. Non-adaptive strategies take up the blame of oneself, which is the fact of taking responsibility for the lived event, contrary to the blame of others, where the responsibility will be attributed to the other. The ruminating of focusing on the feelings and thoughts associated with the lived event and dramatization, when the individual explicitly emphasizes the negative aspects of the lived event.

Studies have highlighted the relationship between emotion regulation and cognitive distortions. Gratz and Roemer (2004) showed that people suffering from PTSD had difficulty regulating their own emotions during an experience of emotional distress. Bardeen's (2013) study suggests an association between difficulties related to cognitive regulation of emotions and symptoms of PTSD. Other studies show that emotional regulation strategies in subjects with PTSD are generally unsuitable (Foa, 2007, Fairholme et al., 2013). However, few studies have investigated the link between cognitive distortions and cognitive regulation of emotions (for review Joorman \& Stanton, 2016). However, it seems that individuals who have been exposed to a traumatic event and suffer from PTSD have an alteration of the processes of regulation of emotions and produce cognitive distortions. The interest of this study is therefore to establish the link between cognitive distortions and cognitive regulation of emotions within a pathological population.

To our knowledge, few studies have been completed with possible interactions between cognitive regulation of emotions and cognitive distortion in a population reported by PTSD (Lau, Pettit \& Creswell, 2013). This study has several main objectives, like to link the production of cognitive distortions with emotion regulation strategies. The second is to determine that there is a profile of ESPT subjects regarding these two dimensions. The goal of this research is spreading beyond the positive impact of the specific profile of cognitive distortions and cognitive regulation of emotions. A profile that would not be present in exposed subjects without PTSD.

\section{Method \& protocol}

One hundred and eighty-three subjects divided into three groups participated in our study. A group with PTSD following exposure to a traumatic event $(n=60)$, a group of subjects exposed to a traumatic event but who did not develop PTSD $(n=63)$ and a control group $(n=63)=61)$ to identify possible differences between the 3 groups.

The PCL-5 (Weathers, 2013) is a 20-item self-administered questionnaire that assesses the 20 symptoms of PTSD according to DSM 5. The PCL-5 has several goals, including assessing changes in symptoms before and after treatment, screening of individuals with PTSD, and the provisional diagnosis of PTSD. Responses are rated from 0 (not at all) to 4 (extremely).

The assessment scale of adult cognitive distortions of Robert, Combalbert, Pennequin, Deperrois $\&$ Ouhmad (2018) was validated on a population of 916 French subjects ( 765 women and 151 men) aged 18 to $85(\mathrm{M}=33.9, \mathrm{SD}=14.8)$. This scale includes 42 mini-scenarios on everyday life based on cognitive distortions presented in Franceschi's (2007) model. Each scenario is accompanied by a proposal of what one might think in a similar situation. The response proposals correspond to cognitive distortions. The distortions are divided into 7 subscales (dichotomous reasoning, omission of the neutral, disqualification of one of the poles, arbitrary focus, maximization, minimization, requalification in the other pole) each comprising 6 scenarios. Three suggest "positive" valence distortion, 3 suggesting "negative" valence distortion. The subjects must assign a score ranging from 0 (does not correspond at all to what I think) to 10 (corresponds quite to what I think). A high score signifying the subject's adherence to distortion.

The aim of the Cognitive Emotion Regulation Questionnaire, developed by Garnefski, Kraaij and Spinhoven (2001), is to evaluate the cognitive and emotional regulation capacity of individuals. It contains 36 items and was developed and validated in its French version (Jermann et al., 2006). This tool measures the preferential production of emotional regulation strategies about what people think and not what they do. Each of the strategies listed below is targeted by 4 different items.

This research was conducted in compliance with the Code of Ethics (1996 updated in February 2012). Participation was free, entirely voluntary and anonymous. The results have been submitted to statistical studies, respecting the confidentiality of each one. The participants were informed that they could stop their participation in the study and that they had access to give them, they also received no bonuses and they signed a free and informed consent form. 
All three groups were split according to the PCL-5 score. The data was analyzed using the Statistica software (Version 13). One-way ANOVAs (Analysis of Variance) were conducted to compare our three groups. We also have correlation analyzes between the two variables emotional cognitive regulation and cognitive distortions.

\section{Results}

Figure 1. Differences in cognitive distortions profiles (positive and negative) between the three groups.

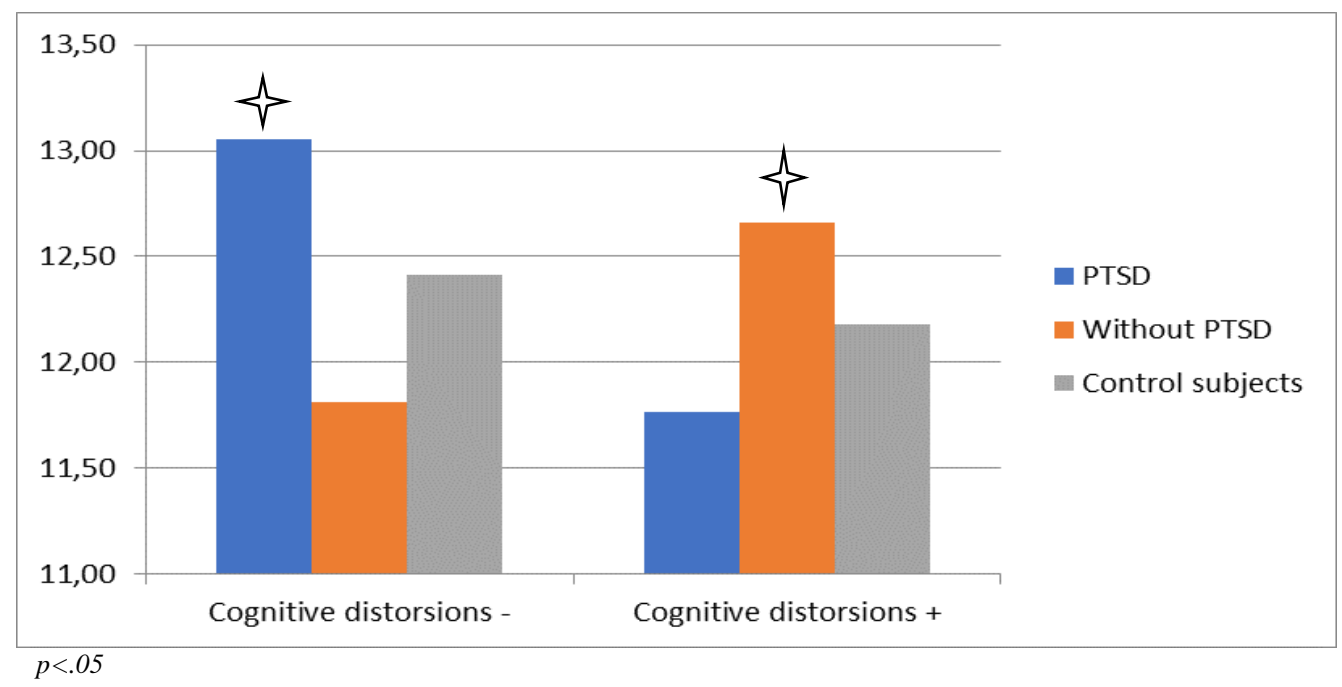

The results show that the group "exposed without PTSD" uses significantly more CD- than CD + . There is also a significant difference between the groups concerning positive cognitive distortions $(\mathrm{F}(2,181)=8,727, \mathrm{p}<.01)$. This is not the case for the negative cognitive distortions $(\mathrm{F}(2,181)=1,985$, $\mathrm{p}>1)$.

Table 1. Differences in non-adaptative emotional regulation between the three groups.

\begin{tabular}{|c|c|c|c|}
\hline & PTSD & Whhout PTSD & $\begin{array}{l}\text { Control } \\
\text { Subjects }\end{array}$ \\
\hline Blâme de soĩ & $10,92(4,85)$ & $8,84(4,45)$ & $10,48(4,44)$ \\
\hline Rumination & $13,45(4,07)^{*}$ & $10,79(4,34)$ & $12,64(4,40)$ \\
\hline Dramatisation & $10,75(4,42)^{*}$ & $8,18(4,01)$ & $7,77(3,55)$ \\
\hline Blâme d'autruĩ & $9,20(4,79)^{*}$ & $9,10(5,28)$ & $7,03(3,35)$ \\
\hline NAR Total & $44,32(12,54)^{*}$ & $36,90(13,16)$ & $37,92(11,75)$ \\
\hline
\end{tabular}

In terms of emotional cognitive regulation, the results show that all groups have significantly higher scores on non-adaptive regulation. Significant results are noted notably for rumination

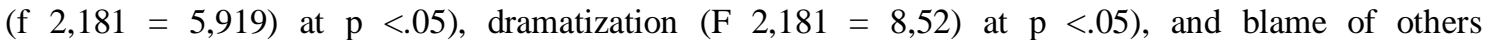
$(\mathrm{F} 2,181=4,67)$ at $\mathrm{p}<.05)$.

\section{Discussion}

The main objective of the study was to verify the presence of specific profile in people with PTSD. The results do suggest the existence of this specific profile concerning cognitive distortions and emotional cognitive regulation in individuals with post-traumatic stress disorder. 
One the one hand, we hypothesized that PTSD would use more negative cognitive distortions because we thought they would be more likely to blame themselves and feel responsible. According to Chung et al., (2014), the characteristics of a trauma can influence the management techniques of the traumatic event as well as its consequences. It is at this point that individuals will negatively evaluate events and thus develop erroneous thought patterns. The results of our study seem to invalidate this postulate. Although individuals with PTSD have a particular profile of cognitive distortions compared to other groups, they seem to be moving in the opposite direction. Indeed, the individuals ESPT seem to use more positive distortions and less negative distortions than the other two groups. These results can be explained by the fact that ESPT subjects benefit from a therapeutic follow-up (psychologist / psychiatrist and / or drug treatment) directly related to developed PTSD.

One the other hand, and consistent with previous studies, we hypothesized that PTSD would use more non-adaptive emotional regulation strategies than the other two groups. The results confirm our hypothesis. Indeed, PTSD is positively associated with strategies of emotional avoidance and control (Roemer et al., 2001, Bardeen et al., 2015) but also with regulatory strategies considered appropriate (Aldao et al. 2010, Webb et al., 2012). In addition, the results show that ESPT individuals preferentially use non-adaptive strategies such as rumination, dramatization, and blaming others. This suggests that PTSD individuals are more likely to blame themselves than others. Our results therefore suggest that people exposed to trauma who developed PTSD would have a particular profile of cognitive distortion production. Indeed, it seems that they produce more positive valence cognitive distortions. In addition, it appears that ESPT subjects use cognitive regulation strategies for inappropriate emotions.

Despite the observations made, it is pertinent to consider the practical and methodological limits of this research. Concerning the protocol: first of all, subjects do not have a conscious access to all the strategies they use, be they cognitive distortions or strategies of regulation of emotions. These are not strategies that subjects voluntarily put in place. Second, the form of the handover (in-line or face-to-face) may be biased. Indeed, only the subjects ESPT were received by the experimenter in order to pass the protocol. For the sake of social desirability, they have been able to minimize or manage some of their emotions. On the other hand, the control and exposed groups without PTSD responded to the online protocol; again, biases such as social desirability may occur. All of these biases are difficult to control. Concerning the population: ESPT subjects are followed by a professional (psychologist or psychiatrist) and can have a treatment directly related to the trauma. Moreover, follow-ups and treatments can significantly reduce the symptomatology of the trauma.

In order to refine this study, it seems interesting to control as much as possible the impact of a possible therapeutic follow-up and a drug treatment on the individuals. By comparing for example, the effects of therapies: type of follow-up, drug treatment and the combination of both on the regulation of emotions and / or cognitive biases. One could see, for example, what types of bias these methods of care take first.

\section{References}

Aldao, A., Nolen Hoeksema, S \& Schweizer, S. (2010). Emotion-regulation strategies across psychopathology: A meta-analytic review. Clinical Psychology Review, 20, 217-237.

American Psychiatric Association. (2013). Diagnostic and statistical manual of mental disorders (5th edition text revision). Washington, DC: American Psychiatric Publishing.

Bardeen, J. R., Tull, M. T., Stevens, E. N., \& Gratz, K. L. (2015). Further investigation of the association between anxiety sensitivity and posttraumatic stress disorder: Examining the influence of emotional avoidance. Journal of Contextual Behavioral Science, 4, 163-169.

Chung MC, Jalal S, Khan NU. Posttraumatic stress disorder and psychiatric comorbidity following the 2010 flood in Pakistan: Exposure characteristics, cognitive distortions, and emotional suppression. Psychiatry 2014; 77:289-304.

Fairholme CP, Nosen EL, Nillni YI, Schumacher JA, Tull MT, Coffey SF. Sleep disturbance and emotion dysregulation as transdiagnostic processes in a comorbid sample. Behav Res Ther. 2013;51: 540-546.

Franceschi, P. (2007). Compléments pour une théorie des distorsions cognitives. Journal de Thérapie Comportementale et Cognitive, pp. 84-88.

Franceschi, P. (2008). Théorie des distorsions cognitives: application à l'anxiété généralisé. (Elsevier, Ed.) Journal de Thérapie Comportementale et Cognitive, 18, pp 127-131.

Foa EB, Hembree EA, Rothbaum BO. Prolonged Exposure Therapy for PTSD: Emotional Processing of Traumatic Experiences - Therapist Guide. Oxford, England: Oxford University Press, 2007. 
Garnefski, N., Kraaij, V., \& Spinhoven, P. (2001). Negative life events, cognitive emotion regulation and emotional problems. Personality and Individuals Differences, 30, pp. 13111327.

Gratz, K. L., \& Roemer, L. (2004). Multidimensional assessment of emotion regulation and dysregulation: Development, factor structure, and initial validation of the Difficulties in Emotion Regulation Scale. Journal of Psychopathology and Behavioral Assessment, 36, 41-54.

Gross, J.J. (2001). Emotion regulation in adulthood: Timing is everything. Current Directions in Psychological Science, 10, pp 214-219.

James, W. (1890). The principles of Psychology. New York : Henry Holt.

Jermann, F., Van der Linden, M., D'Acremont, M. \& Zermatten, A. (2006). Cognitive Emotion Regulation Questionnaire (CERQ): Confirmatory factor analysis and psychometric properties of the french translation. European Journal of Psychological Assesment, 22, pp. 126-131.

Joormann, J. \& Stanton, C.H. (2016). Examining emotion regulation in depression: A review and future directions. Behav Res Ther, 86, pp. 35-49.

Lau, J.Y.F., Pettit, E. \& Creswell, C. (2013). Reducing children's social anxiety symptoms: exploring a novel parent-administered cognitive bias modification training intervention. Behav Res Ther, 51, pp. 333-337.

Ochsner, K.N. and Gross, J.J. (2005) The cognitive control of emotion. Trends in Cognitive Sciences, 9, 242-249.

Webb, T. L., Miles, E., \& Sheeran, P. (2012). Dealing with feeling: A meta-analysis of the effectiveness of strategies derived from the process model of emotion regulation. Psychological Bulletin, 138, 775-808 